\title{
Short communication: Enzymatic perspective of galactosidases reveals variations in lactose metabolism among Lactococcus lactis strains
}

\author{
Yu Yang, ${ }^{1}$ Nan $\mathrm{Li}^{2}{ }^{2}$ Yang Jiang, ${ }^{1}$ Zhenmin Liu, ${ }^{2 *}$ Xiaoming Liu, ${ }^{3,4 *}$ Jianxin Zhao,,${ }^{1,3,4}$ Hao Zhang, ${ }^{1,3,5}$ \\ and Wei Chen ${ }^{1,3,5,6}$ \\ ${ }^{1}$ State Key Laboratory of Food Science and Technology, Jiangnan University, Wuxi, Jiangsu 214122, China \\ ${ }^{2}$ State Key Laboratory of Dairy Biotechnology, Shanghai Engineering Research Center of Dairy Biotechnology, Dairy Research Institute, \\ Bright Dairy and Food Co. Ltd., Shanghai 200436, China \\ ${ }^{3}$ School of Food Science and Technology, Jiangnan University, Wuxi, Jiangsu 214122, China \\ ${ }^{4}$ International Joint Research Laboratory for Probiotics, Jiangnan University, Wuxi, Jiangsu 214122, China \\ ${ }^{5}$ National Engineering Research Centre for Functional Food, Wuxi, Jiangsu 214122, China \\ ${ }^{6}$ Beijing Innovation Centre of Food Nutrition and Human Health, Beijing Technology and Business University, Beijing 100048, China
}

\section{ABSTRACT}

To date, most studies of lactose utilization have focused on the genetic diversity of lactic acid bacteria or its influence on product quality, but phenotypic evaluation has rarely been based on metabolic characteristics. In the present study, we investigated the growth, acid production, $\beta$-galactosidase, and 6-phospho- $\beta$ galactosidase activities of 16 Lactococcus lactis strains obtained from various habitats with lactose as the sole carbon source. The $15 \mathrm{~L}$. lactis strains obtained from various habitats exhibited significant differences in growth and acid production characteristics in the de Man, Rogosa, and Sharpe-lactose broth, and 4 strains consumed more lactose when cultured in skim milk than the type strain ATCC 19435. Among these strains, DQHXNQ38-12 mainly produced acetoin and diacetyl when cultured in skim milk, whereas the strains 15M2 and 5G2 produced high levels of acid and formed curd rapidly. We concluded that the use of lactose is necessary for strain adaptation to the dairy niche. Comprehensive studies of lactose use and the fermentation characteristics of L. lactis are of significant importance. Key words: lactose utilization, Lactococcus lactis, galactosidase, fermentation characteristic

\section{Short Communication}

Lactococcus lactis is traditionally used as a starter culture in the production of fermented dairy food products, such as cheese, sour cream, and buttermilk (Cavanagh et al., 2015). Two main pathways of lactose uptake and metabolism have been described for L. lactis. In the

Received November 11, 2018.

Accepted March 13, 2019.

*Corresponding authors: liuzhenmin@brightdairy.com and liuxm@ jiangnan.edu.cn first pathway, during uptake, lactose is phosphorylated via the lactose phosphotransferase system to lactose6 -phpsphate, which is then hydrolyzed into galatose6 -phosphate and glucose by phospho- $\beta$-galactosidase. Galatose-6-phosphate is then fermented to lactic acid via the tagatose-6-phosphate (T6P) pathway. In the second pathway, lactose is internalized into the cell via a specific permease on the cell membrane and is then cleaved to galactose and glucose by $\beta$-galactosidase; galactose then enters the Leloir pathway (Solopova et al., 2012). Studies have suggested that the Leloir pathway of lactose metabolism normally occurs in the genome of lactic acid bacteria (LAB) whereas the T6P pathway is species specific in L. lactis (Wu et al., 2015). This finding indicates that the lactose metabolism of $L$. lactis strains may differ within species.

$\beta$-Galactosidase and 6 -phospho- $\beta$-galactosidase are the key enzymes of lactose metabolism in LAB (Lansky et al., 2014; Wu et al., 2015), and lactose metabolism has been linked to the formation of flavor compounds, such as diacetyl, acetoin, acetaldehyde, and acetic acid, via a pyruvate intermediate (Smit et al., 2005). However, few studies have investigated the variations in the activities of these enzymes and the use of lactose across L. lactis strains and their correlation with fermentation properties.

In this study, we used the $16 \mathrm{~S}$ rDNA approach to identify 16 strains of $L$. lactis isolated from various sources, including human feces, fermented vegetable, and dairy products (Supplemental Table S1; https:// doi.org/10.3168/jds.2018-15973), such as fermented yak milk and dairy fan - a unique traditional cheese-like dairy product produced in the Yunnan province (Liu et al., 2009). To test the lactose usability of the strains, de Man, Rogosa, and Sharpe (MRS)-lactose broth with lactose as the sole carbon source was prepared by replacing glucose in the medium with lactose $(\mathrm{Wu}$ and Shah, 2017). The strains were cultivated in the MRS- 
lactose broth at $30^{\circ} \mathrm{C}$ under aerobic conditions, and their growth and acid production characteristics were compared including a type strain ATCC 19435. Fermentation characteristics of 8 selected strains (ATCC 19435, MA14, 15M2, 5G2, 6G5, DQHXNQ38-12, ZN8, and D-XJ4-12) that showed the highest growth and acid production were further studied in reconstituted skim milk. The cell-free supernatants of these $8 \mathrm{~L}$. lactis strains after $12 \mathrm{~h}$ of incubation in skim milk were analyzed for sugar metabolism, and the $\beta$-galactosidase and 6-phospho- $\beta$-galactosidase activities were measured as previously described (Wu and Shah, 2017). Gas chromatography-mass spectrometry analysis of the compounds produced in fermented milk was performed and principal component analysis was used to characterize the strains (Settachaimongkon et al., 2014; Zhao et al., 2016). Significant differences $(P<0.05)$ between the strains were determined by 1-way ANOVA using IBM SPSS Statistics version 21.0 (IBM Corp., Armonk, NY).

The $15 \mathrm{~L}$. lactis strains obtained from various habitats exhibited significant differences in growth and acid production characteristics in the MRS-lactose broth (Figure 1A and 1B) but showed similar growth rates and reached similar cell densities when grown in normal MRS broth (data not shown). Compared with the other strains, the strains MA14, 15M2, DQHXNQ38-12, $5 \mathrm{G} 2$, and 6G5 exhibited significantly higher growth rates with a rapid decrease in the $\mathrm{pH}$ value on culturing in skim milk for 2 to $8 \mathrm{~h}$ and reached the stationary phase after $8 \mathrm{~h}$. All of these strains were isolated from dairy niches, such as fermented yak milk, sour cream, and Qula, a traditional cheese made from yak milk in Qinghai province (Duan et al., 2008). Our data support the theory that niche-specific adaptations that occur in environmental isolates play an important role in the transition of bacterial strains, which previously belonged to another niche, to the dairy niche; for example, the ancestor of L. lactis originally inhabited the plant niche (Siezen et al., 2011; Bachmann et al., 2012). The strains H30G-6, HeNa-21-8-GMM, HeNa28-3-GMM, 15M9, DYNDL61M06, DQHXNQ39M56, DSCAB14M3, and ZN8 exhibited a slow but continuous increase in cell densities and a decrease in $\mathrm{pH}$ during incubation. The strains D-XJ4-12 and DYNDL19-5 exhibited a weaker growth and acid-producing ability than strains 5G2 and 15M2.

In total, 5 strains with relatively rapid acid-producing ability (MA14, 15M2, DQHXNQ38-12, 5G2, and 6G5) were selected for further analysis. Compared with the strains ATCC 19435, ZN8, and D-XJ4-12, which were used as controls, these 5 strains exhibited significantly different growth and titratable acidity when cultured in skim milk (Figure 2A and 2B). The titratable acidity of L. lactis 5G2 and 15M2 obtained from fermented yak milk reached $85^{\circ} \mathrm{T}$ after $12 \mathrm{~h}$, whereas that of ATCC 19435, MA14, ZN8, 6G5, and DQHXNQ38-12 reached $30^{\circ} \mathrm{T}$ to $50^{\circ} \mathrm{T}$ and that of D-XJ4-12 changed only slightly. Therefore, strains that had similar growth rates and cell densities when grown in the MRS-lactose broth demonstrated significantly different fermentation characteristics when grown in skim milk, likely due to the variation in the nutrients such as intact milk protein ( $80 \%$ casein, $20 \%$ whey protein) in skim milk and tryptone in MRS-lactose broth.

The results of residual lactose and galactose contents are shown in Figure 2C. Lactococcus lactis 5G2, 15M2, DQHXNQ38-12, and ZN8 consumed approximately 10 to $16 \%$ of the lactose after $12 \mathrm{~h}$ of fermentation, whereas L. lactis 6G5, MA14, and ATCC 19435 consumed only approximately 5\%, and D-XJ4-12 did not significantly change the lactose content. In addition, the reduction in galactose levels after fermentation varied among the strains, with the most significant reduction in the galactose content shown by ZN8.
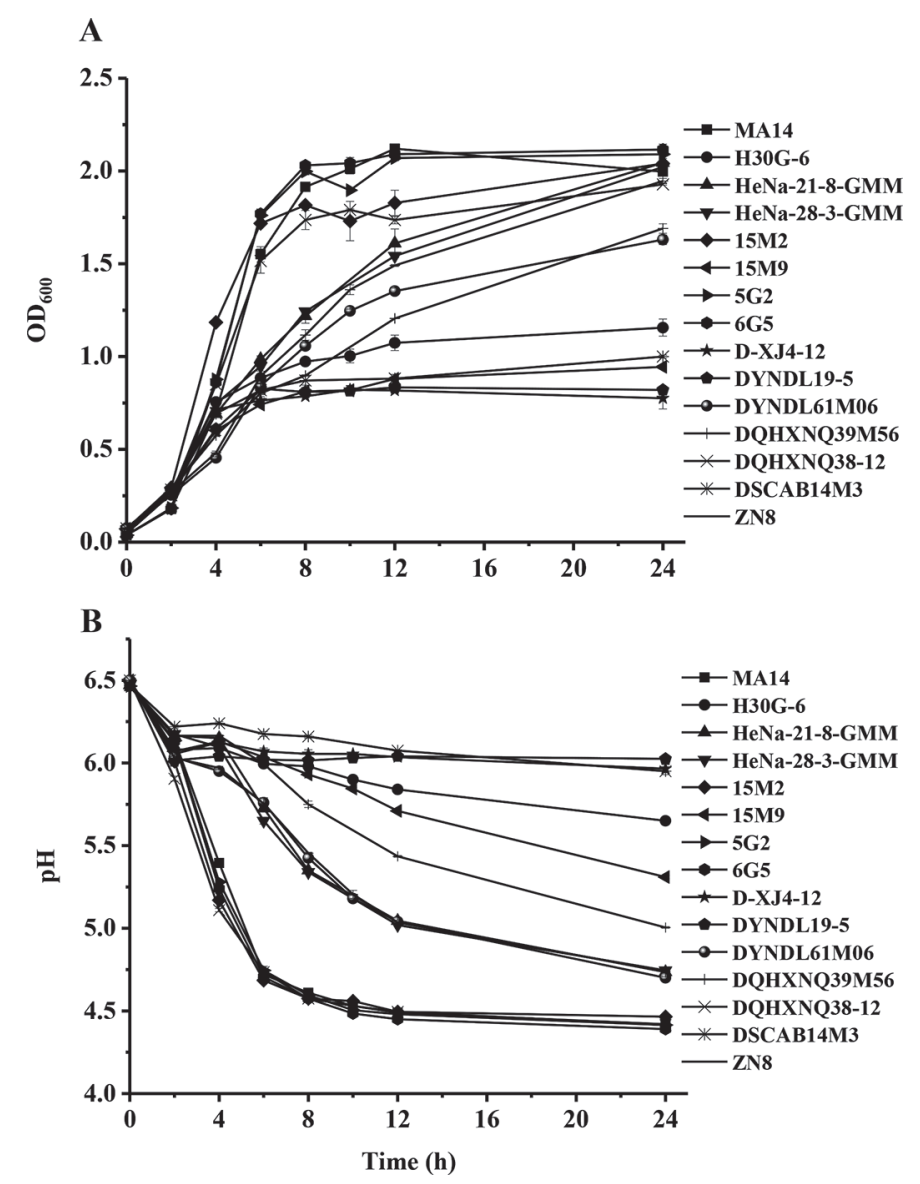

Figure 1. Growth (A) and acid production (B) of Lactococcus lactis in de Man, Rogosa, and Sharpe-lactose broth; growth was measured over $24 \mathrm{~h}$. Growth curves were analyzed in duplicate, and data points represent the average $\pm \mathrm{SE}$. $\mathrm{OD}_{600}=$ optical density at $600 \mathrm{~nm}$. 
It is known that L. lactis NCDO2054 expresses $\beta$-galactosidase to catalyze the intracellular lactose (Vaughan et al., 1998), whereas many other strains, such as L. lactis NCDO712, SK11, and UC509.9, exhibited the T6P pathway for lactose utilization (Gasson, 1983; Wu et al., 2015). Hence, the variation in the use of lactose among $L$. lactis strains was further investigated, and the results are shown in Figure 2D. Lactococcus lactis ATCC 19435, DQHXNQ38-12, 6G5, 5G2, 15M2, and MA14 exhibited 6-P- $\beta$-galactosidase activity, with the highest activity shown by strain ATCC 19435, a type strain isolated from the dairy niche with the complete genes for T6P pathway in the draft genome (Fujii et al., 2015). In a previous study, the 6 -P- $\beta$-galactosidase activity of $65 \mathrm{LAB}$ strains of 15 species, including $L$. lactis, was reported to range from 0 to 50 units (Honda et al., 2007). It has been reported that LAB may obtain the phospho- $\beta$-galactosidase gene via horizontal gene transfer while co-existing with other bacteria, such as Lactobacillus casei, as a previous study suggested that this enzyme is plasmid-encoded in L. lactis and chromosome encoded in L. casei (Wu and Shah, 2017). In contrast, among the 8 strains (ATCC 19435, MA14, 15M2, 5G2, 6G5, DQHXNQ38-12, ZN8, and D-XJ4-12), only $\mathrm{ZN} 8$ expressed $\beta$-galactosidase. This finding is consistent with that of Aleksandrzak-Piekarczyk (2013), who showed that the lactose permease $\beta$-galactosidase system plays a minor role in L. lactis strains. Although the strains exhibiting 6 -P- $\beta$-galactosidase activity were isolated from various dairy niches, we need to be cautious to draw conclusions regarding the connection between ecological niches because the $\beta$-galactosidase
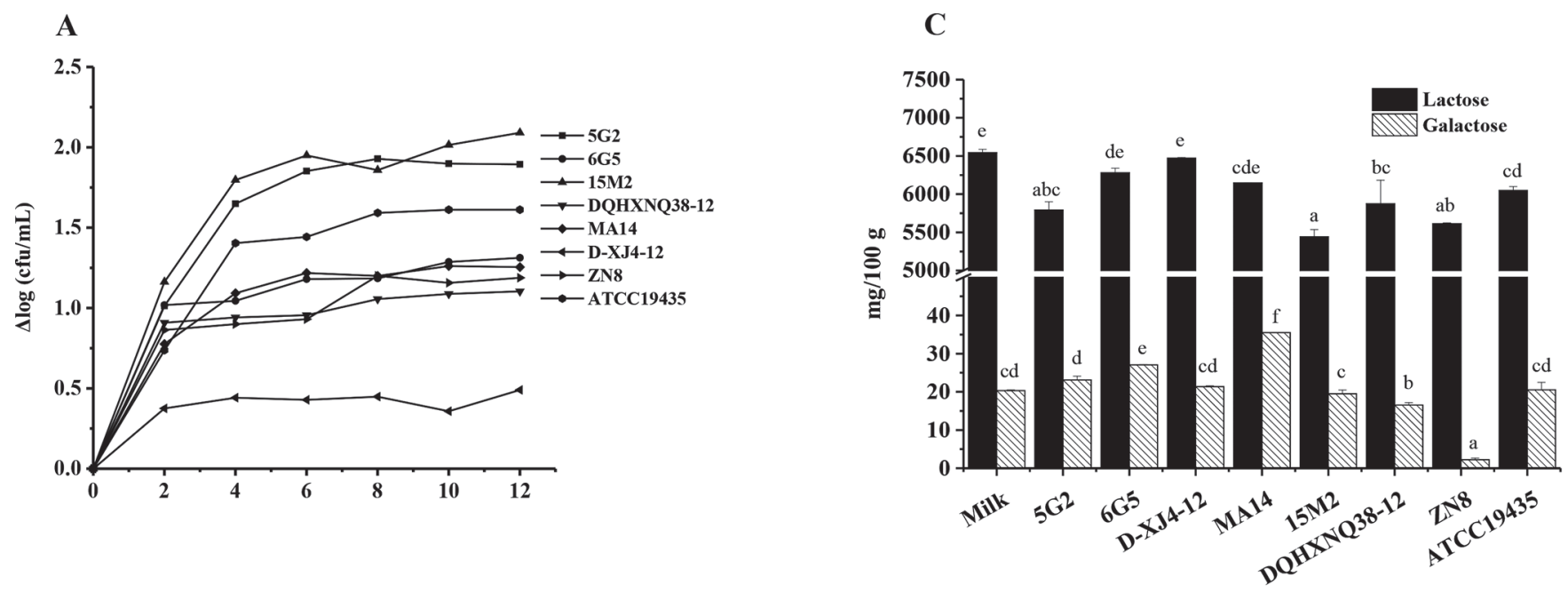

D
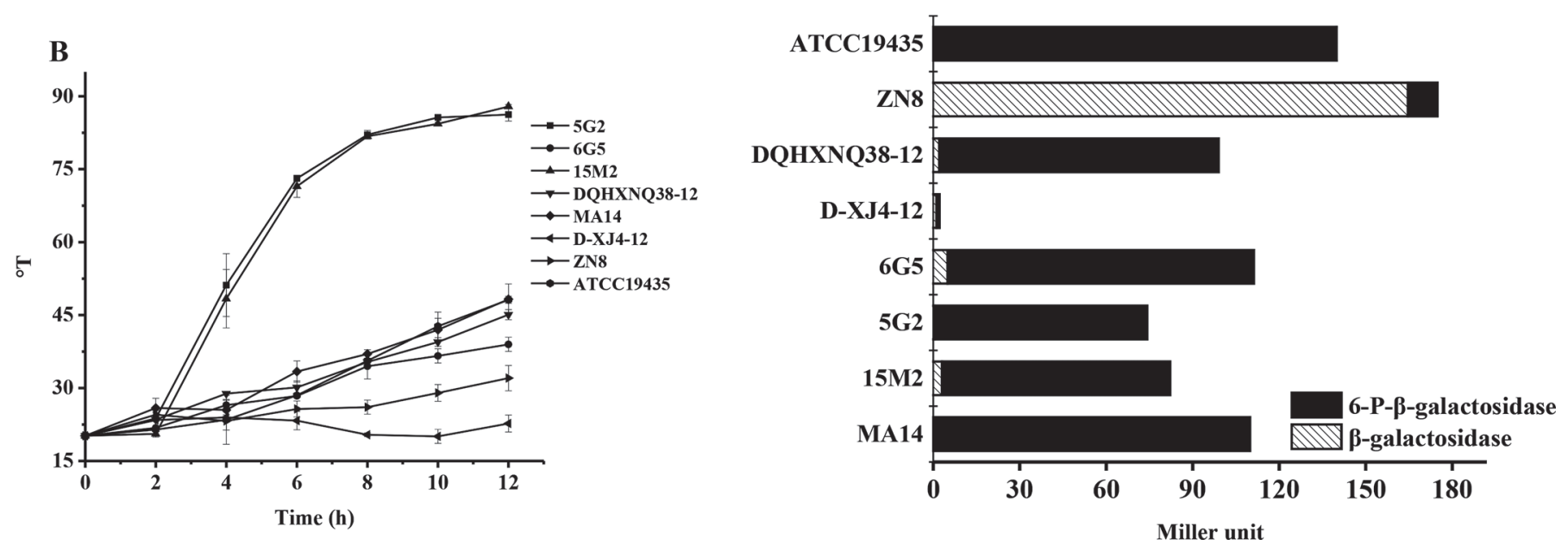

Figure 2. Growth (A) and acid production (B) of Lactococcus lactis in reconstituted skim milk; growth was measured over 12 h. Growth curves were analyzed in duplicate, and data points represent the average \pm SE. (C) Residual lactose and galactose content after cultivation, which was carried out at $37^{\circ} \mathrm{C}$ for $12 \mathrm{~h}$. (D) $\beta$-Galactosidase and 6-phosphate- $\beta$-galactosidase of L. lactis detected within 90 min in the o-nitrophenyl$\beta$-D-galactopyranoside assay. Letters $(\mathrm{a}-\mathrm{f})$ above bars indicate significance: values with no letters in common indicate a significance of $P<0.05$. 
and 6 -P- $\beta$-galactosidase activities were not observed for D-XJ4-12, a strain separated from koumiss. Therefore, classifying the L. lactis strains into domesticated and environmental strains is more appropriate because we do not possess the information of the history of the strains in specific environments (Passerini et al., 2010). In addition, the slow growth of D-XJ4-12 in skim milk may correlate with the lack of enzymatic activities associated with the use of lactose. Taken together, the results indicate variation among $L$. lactis strains in the ability of lactose utilization, via the T6P pathway, via the Leloir pathway, or both.

Figure $3 \mathrm{~A}$ and $3 \mathrm{~B}$ presents the concentrations of typical volatile compounds, including lactic acid, acetic acid, diacetyl, acetoin, and acetaldehyde, considered as the main metabolites of lactose metabolism. The highest concentration of lactic acid was present in the sample of $5 \mathrm{G} 2$, and that of acetic acid was present in the sample of 15M2. However, diacetyl, acetoin, and acetaldehyde, which are desirable compounds in fermented milk, were present in highest concentrations in the sample of DQHXNQ38-12. Lactic acid, acetic acid, and acetaldehyde are the important metabolites of lactose when metabolized via the pyruvate metabolism pathway, and pyruvate can also be metabolized to flavor compounds such as diacetyl and acetoin, which play important roles in the flavor of dairy products (Stefanovic et al., 2017).

The differences in metabolites, fermentation characteristics in skim milk, and the use of lactose revealed by principal component analysis are shown in Figure 3C. Notably, D-XJ4-12 appeared to be isolated from the rest of the strains mainly due to its lack of ability to use lactose. Lactococcus lactis 5G2 was clustered with $15 \mathrm{M} 2$ because they share similar acetic acid and lactic acid production abilities. In contrast, DQHXNQ38-12 showed the highest ketone-producing activity among the strains; it produced the highest levels of acetaldehyde, diacetyl, and acetoin.

Interestingly, 5G2 and DQHXNQ38-12, which showed roughly similar residual lactose contents in skim milk, showed considerably different phenotypic characteristics. Although these 2 strains showed similar lactose metabolism abilities, variations in their metabolic patterns could produce different flavor profiles in fermented dairy products. Our findings highlight the variations in lactose metabolism abilities among 16 strains of $L$. lactis, and half of those strains exhibited different activities of galactosidases with 3 strains (15M2, 5G2, and DQHXNQ38-12) having potential in application in dairy fermentation. It also suggests the requirement of further whole-genome sequence analysis to reveal the genetic basis of different phenotypic characteristics among the 8 strains that had significance variation
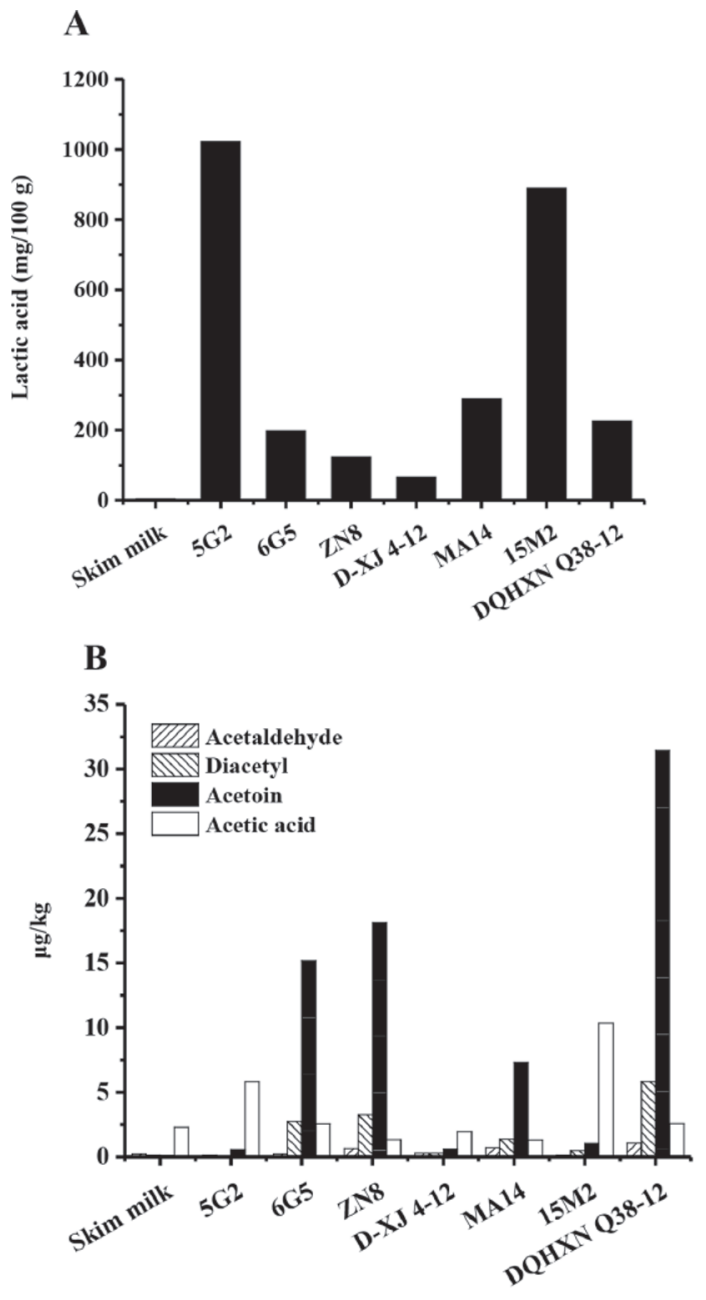

C

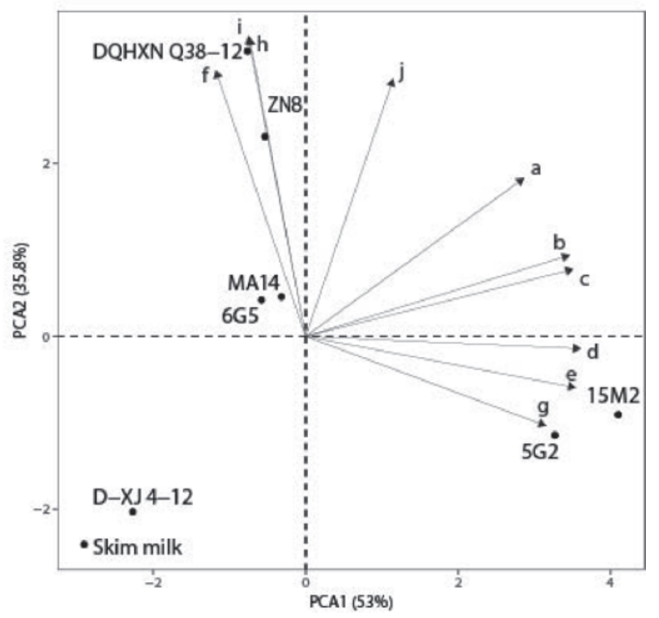

Figure 3. (A) Lactic acid production. (B) Acetaldehyde, diacetyl, acetoin, and acetic acid production. (C) Principal component analysis (PCA) plots of compounds for which significant difference was observed according to LSD test, produced by 7 Lactococcus lactis incubated for $12 \mathrm{~h}$ at $37^{\circ} \mathrm{C}$ in skim milk. (a) Lactose utilization, (b) living bacteria count, (c) pH, (d) titration acidity, (e) lactic acid, (f) acetaldehyde, (g) acetic acid, (h) diacetyl, (i) acetoin, and (j) total activity of $\beta$-galactosidases and 6-phospho- $\beta$-galactosidase. 
in galactosidase activities. Evaluation of subsequent metabolic fluxes is also warranted to explain the variations in volatile compound profiles and determine the pathways involved in lactose metabolism, such as the analysis of end products and intermediate metabolites, before application in dairy fermentation.

In summary, this study presents insights into the enzymatic and phenotypic diversities of the 16 strains of L. lactis, and provides evidence to support the importance of lactose metabolism in the adaptation to dairy niche with 8 strains exhibiting a significant difference in galactosidase activities. Analysis of the flavor compounds from lactose metabolism revealed 3 strains that have the potential to be applied in the dairy industry. However, a combined investigation of the genomic and phenotypic traits of those strains could be an efficient strategy to explain the variations and recommend them strongly as starter cultures that can be applied in dairy fermentation.

\section{ACKNOWLEDGMENTS}

The present research work was supported by the National Natural Science Foundation of China (no. 31871829 and 31371803), Agricultural Science and Technology Innovation Fund of Jiangsu Province [CX(17)1005; China], National First-Class Discipline Program of Food Science and Technology (JUFSTR20180102; China), and Collaborative Innovation Centre of Food Safety and Quality Control in Jiangsu Province (China).

\section{REFERENCES}

Aleksandrzak-Piekarczyk, T. 2013. Lactose and $\beta$-Glucosides metabolism and its regulation in Lactococcus lactis: A Review. Pages 467-486 in Lactic Acid Bacteria - R \& D for Food, Health and Livestock Purposes. Chapter 20. J. Marcelino, ed. IntechOpen. https://doi.org/10.5772/50889.

Bachmann, H., M. J. Starrenburg, D. Molenaar, M. Kleerebezem, and J. E. van Hylckama Vlieg. 2012. Microbial domestication signatures of Lactococcus lactis can be reproduced by experimental evolution. Genome Res. 22:115-124. https://doi.org/10.1101/gr .121285 .111 .

Cavanagh, D., G. F. Fitzgerald, and O. McAuliffe. 2015. From field to fermentation: The origins of Lactococcus lactis and its domestication to the dairy environment. Food Microbiol. 47:45-61. https:// doi.org/10.1016/j.fm.2014.11.001.

Duan, Y., Z. Tan, Y. Wang, Z. Li, Z. Li, G. Qin, Y. Huo, and Y. Cai. 2008. Identification and characterization of lactic acid bacteria isolated from Tibetan Qula cheese. J. Gen. Appl. Microbiol. 54:51-60. https://doi.org/10.2323/jgam.54.51.

Fujii, T., Y. Tomita, S. Ikushima, A. Horie, and D. Fujiwara. 2015. Draft genome sequence of Lactococcus lactis ssp. lactis JCM $5805 \mathrm{~T}$, a strain that induces plasmacytoid dendritic cell activation. Genome Announc. 3:e00113-e00115. https://doi.org/10 .1128/genomeA.00113-15.
Gasson, M. J. 1983. Plasmid complements of Streptococcus lactis NCDO 712 and other lactic streptococci after protoplast-induced curing. J. Bacteriol. 154:1-9.

Honda, H., F. Kataoka, S. Nagaoka, Y. Kawai, H. Kitazawa, H. Itoh, K. Kimura, N. Taketomo, Y. Yamazaki, Y. Tateno, and T. Saito. 2007. Beta-galactosidase, phospho-beta-galactosidase and phospho-beta-glucosidase activities in lactobacilli strains isolated from human faeces. Lett. Appl. Microbiol. 45:461-466. https://doi.org/ 10.1111/j.1472-765X.2007.02176.x.

Lansky, S., A. Zehavi, R. Dann, H. Dvir, H. Belrhali, Y. Shoham, and G. Shoham. 2014. Purification, crystallization and preliminary crystallographic analysis of Gan1D, a GH1 6-phospho- $\beta$ galactosidase from Geobacillus stearothermophilus T1. Acta Crystallogr. F Struct. Biol. Commun. 70:225-231. https://doi.org/10 $.1107 /$ S2053230X13034778.

Liu, W., Z. Sun, J. Zhang, W. Gao, W. Wang, L. Wu, T. Sun, W. Chen, X. Liu, and H. Zhang. 2009. Analysis of microbial composition in acid whey for dairy fan making in Yunnan by conventional method and 16S rRNA sequencing. Curr. Microbiol. 59:199-205. https://doi.org/10.1007/s00284-009-9423-x.

Passerini, D., C. Beltramo, M. Coddeville, Y. Quentin, P. Ritzenthaler, M.-L. Daveran-Mingot, and P. Le Bourgeois. 2010. Genes but not genomes reveal bacterial domestication of Lactococcus lactis. PLoS One 5:e15306. https://doi.org/10.1371/journal.pone.0015306.

Settachaimongkon, S., M. J. Nout, E. C. Antunes Fernandes, K. A. Hettinga, J. M. Vervoort, T. C. van Hooijdonk, M. H. Zwietering, E. J. Smid, and H. J. van Valenberg. 2014. Influence of different proteolytic strains of Streptococcus thermophilus in co-culture with Lactobacillus delbrueckii ssp. bulgaricus on the metabolite profile of set-yoghurt. Int. J. Food Microbiol. 177:29-36. https://doi.org/ 10.1016/j.ijfoodmicro.2014.02.008.

Siezen, R. J., J. R. Bayjanov, G. E. Felis, M. R. van der Sijde, M. Starrenburg, D. Molenaar, M. Wels, S. van Hijum, and J. Vlieg. 2011. Genome-scale diversity and niche adaptation analysis of Lactococcus lactis by comparative genome hybridization using multi-strain arrays. Microb. Biotechnol. 4:383-402. https://doi.org/10.1111/j .1751-7915.2011.00247.x.

Smit, G., B. A. Smit, and W. J. Engels. 2005. Flavour formation by lactic acid bacteria and biochemical flavour profiling of cheese products. FEMS Microbiol. Rev. 29:591-610. https://doi.org/10 .1016/j.femsre.2005.04.002.

Solopova, A., H. Bachmann, B. Teusink, J. Kok, A. R. Neves, and O. P. Kuipers. 2012. A specific mutation in the promoter region of the silent cel cluster accounts for the appearance of lactoseutilizing Lactococcus lactis MG1363. Appl. Environ. Microbiol. 78:5612-5621. https://doi.org/10.1128/AEM.00455-12.

Stefanovic, E., K. N. Kilcawley, M. C. Rea, G. F. Fitzgerald, and O. McAuliffe. 2017. Genetic, enzymatic and metabolite profiling of the Lactobacillus casei group reveals strain biodiversity and potential applications for flavour diversification. J. Appl. Microbiol. 122:1245-1261. https://doi.org/10.1111/jam.13420.

Vaughan, E. E., R. D. Pridmore, and B. Mollet. 1998. Transcriptional regulation and evolution of lactose genes in the galactose-lactose operon of Lactococcus lactis NCDO2054. J. Bacteriol. 180:48934902.

Wu, Q., C. K. W. Cheung, and N. P. Shah. 2015. Towards galactose accumulation in dairy foods fermented by conventional starter cultures: Challenges and strategies. Trends Food Sci. Technol. 41:2436. https://doi.org/10.1016/j.tifs.2014.08.010.

Wu, Q. L., and N. P. Shah. 2017. The potential of species-specific tagatose-6-phosphate (T6P) pathway in Lactobacillus casei group for galactose reduction in fermented dairy foods. Food Microbiol. 62:178-187. https://doi.org/10.1016/j.fm.2016.10.027.

Zhao, N., C. Zhang, Y. Qin, G. Zhuang, Y. Bo, W. Lu, D. Li, F. Tian, X. Liu, and Z. Hao. 2016. Selection of taste markers related to lactic acid bacteria microflora metabolism for Chinese traditional paocai: A gas chromatography-mass spectrometry-based metabolomics approach. J. Agric. Food Chem. 64:2415-2422. https://doi .org/10.1021/acs.jafc.5b05332. 